\title{
Control of Dispersion in Hollow Core Photonic Crystal Fibers
}

\author{
Roberts, John
}

Published in:

Conference on Lasers and Electro-Optics, 2007. CLEO 2007.

Link to article, DOI:

10.1109/CLEO.2007.4453156

Publication date:

2007

Document Version

Publisher's PDF, also known as Version of record

Link back to DTU Orbit

\section{Citation (APA):}

Roberts, J. (2007). Control of Dispersion in Hollow Core Photonic Crystal Fibers. In Conference on Lasers and Electro-Optics, 2007. CLEO 2007. (pp. 1-2). IEEE. https://doi.org/10.1109/CLEO.2007.4453156

\section{General rights}

Copyright and moral rights for the publications made accessible in the public portal are retained by the authors and/or other copyright owners and it is a condition of accessing publications that users recognise and abide by the legal requirements associated with these rights.

- Users may download and print one copy of any publication from the public portal for the purpose of private study or research.

- You may not further distribute the material or use it for any profit-making activity or commercial gain

- You may freely distribute the URL identifying the publication in the public portal

If you believe that this document breaches copyright please contact us providing details, and we will remove access to the work immediately and investigate your claim. 


\title{
Control of Dispersion in Hollow Core Photonic Crystal Fibers
}

\author{
P. J. Roberts \\ Department of Communications, Optics and Materials, Danish Technical University, DK-2800 Kgs. Lyngby, Denmark \\ pjr@com.dtu.dk
}

\begin{abstract}
The dispersion of hollow core photonic crystal fibers can be tailored by modifying a single ring of holes in the cladding. The dispersion can be lowered and flattened, or alternatively greatly increased, in a controlled manner.

2006 Optical Society of America

OCIS codes: (060.2310) Fiber optics; (060.2400) Fiber properties
\end{abstract}

\section{Introduction}

Hollow core photonic crystal fibers (HC-PCFs) offer the possibility of low-loss signal transmission with greatly reduced nonlinearity, since most of the light power propagates in air. The decrease in the intrinsic nonlinearity implies that a lower chromatic dispersion can be tolerated, at a given power level, within a communication system. This implies that the need for dispersion compensation can be reduced, or in some cases, eliminated altogether. HCPCF guide by virtue of a bandgap appearing within the cladding photonic density of states. The bandgap guidance mechanism imposes a generic form to the dispersion profile of the archetypal HC-PCF geometry; the second order dispersion value does attain low values within the guidance bandwidth, but is accompanied by a high degree of higher-order dispersion which will degrade a short pulse over a significant propagation length.

Many other applications of HC-PCF would also benefit from modification of the dispersion profile over a broad bandwidth range. A gas can be introduced into the core of the fiber for nonlinear or quantum-optical applications, such as Raman-based frequency generation and electromagnetic induced transparency [1]. High power solitonic pulses of short duration can be propagated within the core of the HC-PCF [2]. The power threshold for their formation and the soliton decay properties are to a large extent dictated by the mode dispersion properties. An important use of HC-PCF is for high power short pulse compression [3], for which high second order dispersion (GVD) is required. The higher order dispersion terms which accompanies a high GVD, however, imposes a lower limit to the attainable pulse duration.

The band-gap guided mode dispersion profile can become modified by an anti-crossing event with another mode of compatible symmetry [4,5]. In fact, one or more such mode interactions generally does occur, within the bandgap range, between the core mode of interest and modes associated with the glass region which surrounds the core and joins onto the cladding. These interactions lead to very high and uncontrolled dispersion in their vicinity and a concomitant increase in loss. To be useful for dispersion tailoring over a broad bandwidth in a controlled manner, an anti-crossing event must involve modes with similar group velocity as well as effective index values. It should also not greatly increase the fiber loss and nonlinearity over the useful waveband. These constraints preclude use of an anti-crossing of the signal mode with a mode associated with core surround, but they can be fulfilled if a subsidiary core with a high effective air filling fraction is introduced into the fiber. The philosophy is directly analogous to dispersion tailoring schemes which have been exploited in conventional TIR fibers, both for dispersion lowering and for achieving high GVD and controlled higher order dispersion over a broad waveband.

\section{Results and discussion}

The essential physics behind the dispersion control of HC-PCF is encapsulated by a simple Bragg-fiber model wherein the refractive index is assumed to be dependent purely on the radial distance from the core center, see Fig. 1(a) and 1(b). The subsidiary core is ascribed a refractive index a little above unity to take account of the inevitable connecting glass struts in the HC-PCF geometry. The model can be used to obtain an approximate subsidiary core size which will become phase matched with the $\mathrm{HE}_{11}$-like mode of the central core in or near the bandgap, and to quickly explore what forms dispersion profiles are likely to be achievable. It does, however, miss the mode anticrossing events with core surround modes which are an important feature associated with of the HC-PCF geometry.

Having narrowed the parameter space with the simplified model, detailed mode-solving of the full HC-PCF geometry with a realistic subsidiary low-index core can be performed. Fig 1(c) shows a practical HC-PCF geometry which is the analogue of the Bragg fiber shown in Fig. 1(a). Superimposed is a typical mode intensity profile calculated for the $\mathrm{HE}_{11}$-like guided mode. The subsidiary core is placed adjacent to the core surround glass with a view to obtaining a low and flattened dispersion. Figure 1(d) shows the dispersion calculated for the fiber forms shown in Fig. 1(a) and 1(c), together with the dispersion profile calculated for the archetypal HC-PCF geometry in 


\section{CWF2.pdf}

which the subsidiary core is replaced by conventional bandgap cladding. It can be seen that, between wavelengths $=1.5 \mathrm{~m}$ and $=1.65 \mathrm{~m}$, the dispersion has been substantially lowered by inclusion of the subsidiary core. Computational time precluded a more thorough investigation of the full dual-core HC-PCF geometry, but it likely that the dispersion can be further reduced and flattened by geometrical refinement.
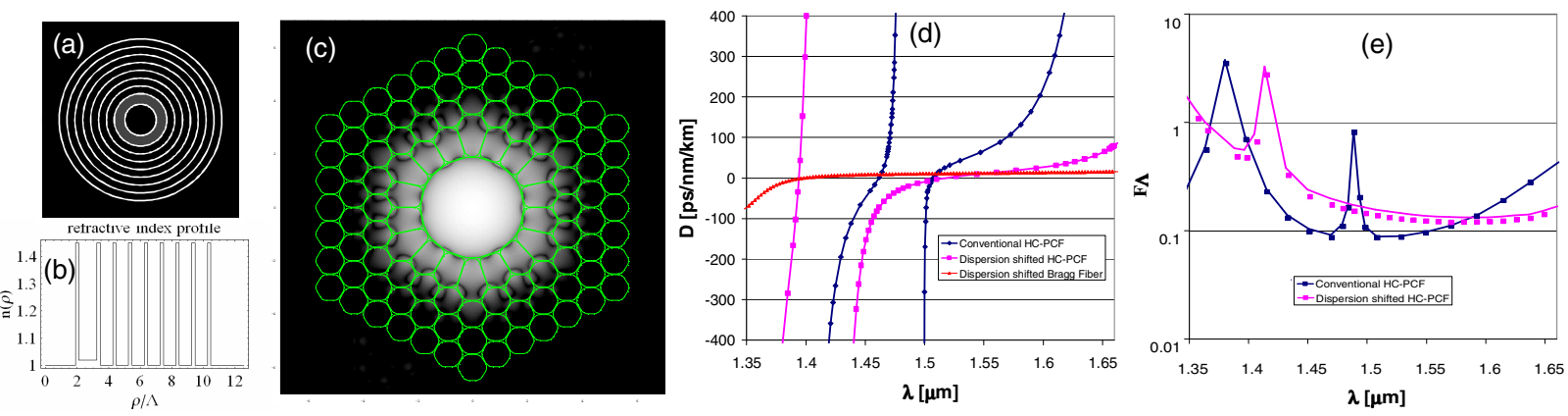

Fig.1 (a) Bragg fiber model for dispersion control. (b) Refractive index profile of Bragg fiber model. (c) The dispersion shifted HCPCF structure. Also shown superimposed is a mode field intensity (log-scale) over a 60dB range. The amount of "spillage" of the field into the ring of holes adjacent the central core is wavelength dependent and enables the dispersion control. (d) Dispersion plot for a conventional HC-PCF (blue), the dispersion shifted form (pink) and the Bragg model fiber (red). (e) The normalized interface field intensity (an approximate relative measure for loss) for the conventional HC-PCF (blue) and the dispersion shifted form (pink)

For the fiber to be useful, the loss in the dispersion-reduced wavelength range should not be substantially increased. Figure 1(e) shows the normalized interface field intensity F for the dual-core and conventional HC-PCF forms. F provides an approximate relative measure of loss [6]. The wavelength dependence of the power in glass fraction and nonlinearity for the two fibers is similar to that shown by F. The loss and nonlinearity is expected to increase by a factor of around 2 for the dispersion-tailored form.

The mode anti-crossing between the $\mathrm{HE}_{11}$-like mode and modes associated with the subsidiary core in fact occurs close to the short wavelength bandgap edge. The peak near $=1.41 \mathrm{~m}$ in the F-trace (pink curve in Fig. 1(e)) for the dispersion shifted HC-PCF is actually related to an anti-crossing with a core-surround mode, but its influence on the dispersion is short-ranged and the subsidiary core is needed to achieve the broadband dispersion lowering and flattening. The long-range interaction between modes associated with the two cores is responsible for the overall trend for $\mathrm{F}$ to increase as the wavelength is reduced. As the subsidiary core is made larger than shown in Fig. 1(c), a myriad of states associated with it encroach further into the bandgap from the blue side and the useable bandwidth is reduced.

To attain large dispersion with a controllable slope, the subsidiary core should be placed further from the central air core, thus better emulating the conventional dispersion compensating fiber form. At the time of writing, for the studied test geometries, the subsidiary core is found to introduce a substantial number of unwanted modes into the band gap which limits the useable bandwidth at high GVD (and low higher order dispersion) to less than about 30 $\mathrm{nm}$. By further tailoring the geometry, it is nevertheless expected that the dispersion can be controlled at a high value over a bandwidth suitable for pulse compression to below $100 \mathrm{fs}$.

\section{Conclusions}

The dispersion of hollow core photonic crystal fibers can be tailored by modifying a single ring of holes in the cladding. The dispersion can be lowered and flattened, in a controlled manner, by enlarging the first ring of cladding holes. High second order dispersion, with reduced higher order dispersion terms, can result if the subsidiary core is placed further from the central air core, but the incursion of unwanted mode interactions is likely to limit the useable bandwidth.

\section{References}

[1] F. Benabid, F. Couny, J. C. Knight et al., "Compact, stable and efficient all-fibre gas cells using hollow-core photonic crystal fibres," Nature 434, 488-491 (2005).

[2] D. G. Ouzounov, F. R. Ahmad, D. Muller et al, "Generation of Megawatt Optical Solitons in Hollow-Core Photonic Band-Gap Fibers," Science 301, 1702-1704 (2003).

[3] C. J. S. de Matos, J. R. Taylor, T. P. Hansen et al, "All-fiber chirped pulse amplification using highly dispersive air-core photonic band gap fiber," Opt. Express 11, 2832-2837 (2003).

[4] J. A. West, C. M. Smith, N. F. Borrelli et al, "Surface modes in air-core photonic band-gap fibers", Opt. Express 12, 1485-1496 (2004).

[5] K. Saitoh, N. A. Mortensen and M. Koshiba, "Air-core photonic band-gap fibers: the impact of surface modes," Opt. Express 12, 394-400 (2004).

[6] P. J. Roberts, F. Couny, H. Sabert et al, "Ultimate low loss of hollow-core photonic crystal fibers", Opt. Express 13, 236-244 (2005). 